\title{
Los rasgos expresivos e instrumentales de la comunidad educativa española: estudiantes, padres/ madres y docentes
}

\section{Instrumental and expressive traits of the spanish community education: students, parents and teachers}

\author{
Yolanda Rodríguez Castro*1, María Lameiras Fernández*, \\ María José Magalhães** \& María Victoria Carrera Fernández* \\ *Universidad de Vigo; Facultad de Ciencias de la Educación, Campus Ourense, España \\ ** Facultad de Psicología e de Ciencias da Educaçao; Universidad de Oporto, Portugal
}

(Recepción: Mayo 2010 - Aceptación: Octubre 2010)

\begin{abstract}
Resumen
El objetivo de este estudio es analizar los rasgos expresivos e instrumentales de la comunidad educativa española (estudiantes, docentes y padres/madres) y determinar si son los padres y las madres o sus docentes los que ejercen la mayor influencia en la transmisión de los estereotipos de género de los estudiantes de educación secundaria. Para ello contamos con tres muestras: de docentes no universitarios $(n=744)$, de estudiantes $(n=1113)$ y de sus madres y padres $(n=917)$. Se les administró un cuestionario con variables sociodemográficas y el Cuestionario de Atributos Personales (PAQ), (Spence et al., 1974). Los resultados demostraron que las mujeres siguen siendo más Expresivas, mientras que los hombres son más Instrumentales e Instrumentales-Expresivos. Y que son los y las docentes los que asume las características más Expresivas y las más Instrumentales y con ello menos estereotipadas. Por ello, la escuela debe implicar a los padres y madres en las prácticas coeducativas.

Palabras claves: expresividad, instrumentalidad, docente, madres, padres y estudiantes.
\end{abstract}

\begin{abstract}
The aim of this study is to analyze the expressive and instrumental traits of the Spanish Educational Community (students, teachers and parents) and to determine whether fathers and mothers or teachers are who have the greatest influence in the transmission of stereotypes gender to students in secondary education. The sample were of teacher $(n=744)$, students $(n=1113)$ and their mothers and fathers $(n=$ 917). The scales were administered: the Personal Attributes Questionnaire, PAQ (Spence et al., 1974). The results showed that women are being more expressive, whereas men are more Instrumental and Instrumental-Expressive. Furthermore, teachers are more expressive and more instrumental. This is that they are less stereotyped. Therefore, schools should involve parents in co-educational practices.
\end{abstract}

Key words: expressiveness, instrumentality, teachers, mothers, parents and students.

\section{Introducción}

Las primeras aproximaciones que fundamentan el estudio del género se hicieron a través de su operacionalización en los denominados rasgos de masculinidad y feminidad que a caracteriza a los hombres como independientes, asertivos, activos, dominantes en el espacio público en detrimento de las mujeres que las describe desde la dependencia, la sensibilidad, el afecto, relegadas al ámbito

1 Correspondencia dirigida a: Yolanda Rodríguez Castro; Faculty of Education, University of Vigo (Spain); Av. Castelao sn, 32004. Pabellón 2. Campus de Ourense, Ourense (Spain). Tel.: 0034988604257. E-mail: yrcastro@uvigo.es. 
privado/domestico. Estos conceptos masculinidad/feminidad han ido evolucionando desde un modelo clásico unidimensional hasta los modelos multidimensionales.

En el modelo clásico de la Congruencia se considera que la Masculinidad y la Femininidad representaban dos polos opuestos de una única dimensión. Lo que supone asumir un concepto de género unidimensional y bipolar, basándose el origen causal de la bipolarización entre Masculinidad y Feminidad en el dimorfismo biológico entre varones y mujeres (Barberá, 1998).

En un segundo momento, la aportación más significativa la va a representar Bem (1974) con su Modelo de Androginia, que posteriormente será asumido por Spence $(1979,1984)$. Modelo que se propone como alternativa plausible al modelo de congruencia claramente cuestionado. A través del concepto de Androginia se intenta minimizar el determinismo de las características biológicas en la construcción del género, de modo que, independientemente del sexo biológico, las personas podrían desarrollar cualidades de masculinidad o feminidad, y serían precisamente aquellas personas que fueran capaces de desarrollar ambas las que alcanzarían su mayor funcionalidad práctica y con ello un mayor ajuste psicológico. Por tanto el mayor ajuste se daría en aquellos sujetos

Partiendo de ambos modelos, el modelo de Equilibrio (Bem, 1974) y el modelo Aditivo (Spence et al., 1974) cristalizan dos medidas de autoinforme que evalúan los rasgos de personalidad en las que se incluyen dos subescalas separadas de Masculinidad y Feminidad, sin duda las más usadas y mejor estudiadas. Estas son el BSRI, Bem Sex Role Inventory (Bem, 1974) y, el PAQ, Personal Attributes Questionaire (Spence et al., 1974).

Sin embargo, los conceptos de masculinidad y feminidad no han estado exentos de controversia por las dificultades de condensar la complejidad de tales constructos en una única medida psicológica y por otro lado por la vinculación directa con el sexo biológico, así que ya desde 1978 Spence y Helmreich abandonan dichos conceptos por etiquetas más descriptivas como Instrumentalidad y Expresividad, reservando las etiquetas de Masculinidad y Feminidad para describir la identidad de género, más global, al que cada persona se autoadscribe. Una prueba de la inadecuación de utilizar los conceptos de Masculinidad y Feminidad para identificar lo que miden tanto la escala BSRI como el PAQ es, según Spence y Buckner (2000), la ausencia de correlación entre estas escalas con aquellas que miden actitudes hacia los sexos. Aunque Spence y Buckner (2000) subrayan que en caso de que existiera alguna relación deberían ser mutuamente congruentes. Es decir, que si existiese relación los hombres que asumen rasgos más instrumentales serían, a su vez, los que asumirían actitudes más sexistas y por su parte las mujeres que se definen con rasgos expresivos serán las que asumirían las actitudes más sexistas.

El sistema educativo español ha asumido desde las últimas décadas una filosofía basada en la igualdad de oportunidades y la no discriminación por razón del sexo, sin embargo la mayoría de los estudios (Lameiras y Rodríguez, 2002, 2003; Lameiras et al., 2006; Rodríguez, 2006) centrados en estudiantes, los chicos y chicas presentan estereotipos de género y actitudes sexistas. Por ello, el objetivo principal de este estudio es analizar los rasgos expresivos e instrumentales de toda la comunidad educativa española incluyendo a estudiantes, a sus docentes y a sus padres/madres, con la finalidad de determinar si son los padres y las madres o sus docentes los que ejercen la mayor influencia en la transmisión de los estereotipos de género de los estudiantes de educación secundaria.

\section{Método}

\section{Participantes}

En este estudio contamos con tres muestras que representan a la población española de docentes no universitarios, estudiantes y sus madres y padres.

A) Docentes: en este estudio contamos con una muestra de 700 docentes no Universitarios de los cuales un $62.5 \%$ son profesoras y un $37.4 \%$ son profesores, con un rango de edad que va desde los 22 a los 65 años. La media de edad de este colectivo es de 41.3 (SD: 9.9). 
B) Estudiantes: en este estudio contamos con una muestra de 1000 estudiantes de Educación Secundaria Obligatoria, de los cuales un $50.1 \%$ son chicas y un $49.9 \%$ son chicos, con un rango de edad que va desde los 14 a los 18 años, siendo la media de edad de 15.4 (SD: 1.1).

C) Padres/Madres: la muestra de padres y madres la constituyen 917 personas, de los cuales 497 son madres y 420 son padres con un rango de edad que va desde los 30 a los 70 años, siendo la media de edad de 43,3 (SD: 5.5).

\section{Procedimiento}

La aplicación de los cuestionarios se llevó a cabo en 85 centros educativo españoles, de un total de 148 centros contactados, con una tasa de respuesta fue del $57.4 \%$.

El muestreo fue de tipo aleatorio polietápico estratificado por conglomerados -constituidos por agregados de Comunidades Autónomas-, con asignación proporcional y selección aleatoria de comunidades, entidades de población y posteriormente forzada hacia centros educativos y dentro de cada centro educativo se seleccionaron dos aulas se educación secundaria en la que tomaron a los y las estudiantes, a sus docentes y a sus madres y padres. En los centros educativos la recogida de datos a docentes y a estudiantes fue realizado durante el horario académico, mientras que el procedimiento seguido para la recogida de datos de sus padres y madres consistió en darle a cada estudiante dos cuestionarios, uno para el padre y el otro para la madre, de forma que cuando los tuvieran cubiertos los entregaran en el centro educativo.

\section{Instrumentos}

1) Variables sociodemográficas como el género, la opción religiosa, la situación laboral (del padre y de la madre), si ocupan cargos en el centro educativo (los y las docentes).

2) Cuestionario de Atributos Personales (PAQ), (Spence et al., 1974), en la versión española de Vergara y Páez (1993). Con este cuestionario se pretende medir la autoadscripción que hace el sujeto de rasgos asertivos-instrumentales así como de rasgos expresivos-interpersonales. Cada característica del PAQ se presenta en una escala bipolar de cinco puntos, de modo que se pide a los sujetos que indiquen en qué punto (1-5) del continuo se sitúan (ej. 1"nada agresivo" a 5 "muy agresivo"). El PAQ está compuesto por tres escalas: 1. Escala de Instrumentalidad; 2. Escala de Instrumentalidad-Expresividad 3. Escala de Expresividad. La fiabilidad obtenida en este estudio con la muestra de docentes fue en la escala de Expresividad de .79, en la escala de Instrumentalidad de .69, y en la escala de Instrumentalidad-Expresividad fue de .72. En el grupo de los estudiantes, la fiabilidad obtenida en la escala de Instrumentalidad fue de .69, en la escala de Expresvidad de $.70 \mathrm{y}$ en la escala de Instrumentalidad-Expresividad fue de .65. Y finalmente en la fiabilidad del PAQ obtenida en el grupo de madres/padres en el la escala de Expresividad fue de .78, en la escala de Instrumentalidad fue de .72, y en la escala de Instrumentalidad-Expresividad fue de .70.

\section{Resultados}

\section{PAQ y variables sociodemográficas}

En las tres muestras de este estudio se puede constatar que hay diferencias significativas en las tres escalas del PAQ en función de la variable género (ver tabla 1). De forma, que las mujeres (profesoras $(\mathrm{t}=3.04 ; \mathrm{p}<.01)$, madres $(\mathrm{t}=8.01 ; \mathrm{p}<.001)$ y alumnas $(\mathrm{t}=11.2 ; \mathrm{p}<.001)$ puntúan más en la escala de Expresividad mientras que los hombres (profesores $(\mathrm{t}=2.30 ; \mathrm{p}<.05)$, alumnos $(\mathrm{t}=-7.97$; $\mathrm{p}<.001)$ y padres $(\mathrm{t}=-5.52 ; \mathrm{p}<.001)$ en Instrumentalidad y también siguen siendo los hombres (profesores $(\mathrm{t}=-10.3 ; \mathrm{p}<.001)$, alumnos $(\mathrm{t}=-14.8 ; \mathrm{p}<.001)$ y padres $(\mathrm{t}=-12.8 ; \mathrm{p}<.001)$ los que mayores puntuaciones obtienen en la escala de Instrumentalidad-Expresividad (ver tabla 1). 
En relación a la opción religiosa del grupo de los docentes (ver tabla 1), destacar que los/as docentes que se definen como no creyentes, son los que más puntúan en la escala de InstrumentalidadExpresividad $\left(\mathrm{F}_{(2,692)}=5.50 ; \mathrm{p}<.01\right)$. Por su parte, los chicos y las chicas (ver tabla 1$)$ que se definen como no creyentes son los que asumen rasgos más instrumentales $\left(\mathrm{F}_{(2,1033)}=4.05 ; \mathrm{p}<.05\right)$ y rasgos más instrumentales-expresivos $\left(\mathrm{F}_{(2,1000)}=2.68 ; \mathrm{p}<.001\right)$. En cuanto a la opción religiosa del grupo de madres y padres (ver tabla 1) señalar que siguen siendo los padres y madres más expresivos los más devotos $\left(\mathrm{F}_{(2,854)}=8.01 ; \mathrm{p}<.001\right)$ mientras que los instrumentales-expresivos son los se definen más como católicos no practicantes $\left(\mathrm{F}_{(2,849)}=5.85 ; \mathrm{p}<.001\right)$. Estos datos nos indican que la opción religiosa es una variable importante a la hora de determinar los rasgos de personalidad estereotipados por el género.

Tabla 1. Diferencias de medias de las escalas de PAQ en relación a las variables sociodemográficas en las tres muestras (estudiantes, padres/madres y docentes)

\begin{tabular}{|c|c|c|c|c|c|c|c|c|c|}
\hline & \multicolumn{3}{|c|}{ Expresividad } & \multicolumn{3}{|c|}{ Instrumentalidad } & \multicolumn{3}{|c|}{ Expresividad-Instrumentalidad } \\
\hline & Est & $\mathrm{P} / \mathrm{M}$ & Doc & Est & $\mathrm{P} / \mathrm{M}$ & Doc & Est & $\mathrm{P} / \mathrm{M}$ & Doc \\
\hline \multicolumn{10}{|l|}{ Género } \\
\hline Mujer & 3.95 & 3.74 & 3.92 & 3.24 & 3.32 & 3.37 & 2.54 & 2.40 & 2.47 \\
\hline \multirow[t]{3}{*}{ Hombre } & 3.56 & 3.44 & 3.79 & 3.49 & 3.56 & 3.50 & 2.97 & 2.75 & 2.80 \\
\hline & $\mathrm{t}=$ & $\mathrm{t}=$ & $\mathrm{t}=$ & $\mathrm{t}=-$ & $\mathrm{t}=-$ & $\mathrm{t}=-$ & $\mathrm{t}=-$ & $\mathrm{t}=-$ & $\mathrm{t}=-$ \\
\hline & $11.2 * * *$ & $8.01 * * *$ & $3.04 * *$ & $7.97 * * *$ & $5.52 * * *$ & $2.30^{*}$ & $14.8^{* * *}$ & $12.8^{* * *}$ & $10.3^{* * *}$ \\
\hline \multicolumn{10}{|l|}{ Opción Religiosa } \\
\hline \multicolumn{10}{|l|}{ Practicante } \\
\hline No practicante & 3.81 & 3.65 & 3.89 & 3.32 & 3.39 & 3.44 & 2.69 & 3.47 & 2.57 \\
\hline \multirow[t]{4}{*}{ No creyente } & 3.75 & 3.59 & 3.84 & 3.33 & 3.39 & 3.42 & 2.76 & 3.54 & 2.57 \\
\hline & 3.70 & 3.42 & 3.81 & 3.46 & 3.49 & $3.44 \mathrm{~F}_{(2)}$ & 2.87 & 32.69 & $2.71 \mathrm{~F}_{(2,}$ \\
\hline & $\mathrm{F}_{(2,1024)}=$ & $\mathrm{F}_{(2,854)}=$ & $\mathrm{F}_{(2,691)}=$ & $\mathrm{F}_{(2,1033)}=$ & $\mathrm{F}_{(2,852)}=$ & ${ }_{697)}=$ & $\mathrm{F}_{(2,1000)}=$ & $\mathrm{F}_{(2,849)}=$ & ${ }_{692)}=$ \\
\hline & 2.41 & $6.96^{* * *}$ & 1.33 & $4.05^{*}$ & .946 & .101 & $6.56^{* * *}$ & $5.85^{* * *}$ & $5.50 * *$ \\
\hline \multicolumn{10}{|l|}{ Situación } \\
\hline \multicolumn{10}{|l|}{ Laboral } \\
\hline No trabaja fuera & & 3.67 & & & 3.27 & & & 2.42 & \\
\hline \multirow[t]{4}{*}{ Si trabaja fuera } & & 3.54 & & & 3.47 & & & 2.60 & \\
\hline & & $t=$ & & & $t=$ & & & $t=$ & \\
\hline & & $-3.66^{* * *}$ & & & & & & $-5.09^{* * * *}$ & \\
\hline & & & & & $-4.64^{* * *}$ & & & & \\
\hline \multicolumn{10}{|l|}{ Cargo en } \\
\hline Centro & & & 3.81 & & & 3.41 & & & 2.59 \\
\hline No & & & 393 & & & $3.52 \mathrm{t}$ & & & 2.66 \\
\hline \multirow[t]{2}{*}{$\mathrm{Si}$} & & & $\mathrm{t}=$ & & & $\mathrm{t}=$ & & & $\mathrm{t}=$ \\
\hline & & & $-2.66^{* * *}$ & & & $-2.60 * *$ & & & -1.86 \\
\hline
\end{tabular}

Nota: ${ }^{*} \mathrm{p}<.05 ;{ }^{* *} \mathrm{p}<.01 ;{ }^{* * *} \mathrm{p}<.001$; Est: muestra de estudiantes; P/M: muestra de padres y madres; y Doc: Muestra de Docentes 
En el grupo de los y las docentes se han detectado diferencias significativas en la variable de ocupar un cargo en el centro (ver tabla 1), de manera que los/as docentes que sí desempeñan algún cargo en el centro son más expresivos $(\mathrm{t}=-2.66 ; \mathrm{p}<.001)$ y a su vez, más instrumentales $(\mathrm{t}=-2.60$; $\mathrm{p}<.01)$. Además, las mujeres con cargos asumen características más expresivas $(\mathrm{t}=19.6 ; \mathrm{p}<.01)$ frente a los hombres con cargos que asumen características más instrumentales-expresivas $(\mathrm{t}=7.60$; $\mathrm{p}<.01$ ). Sin embargo en el grupo de madres y padres, en la variable relacionada con la situación laboral se detectan diferencias en las escalas del PAQ (ver tabla 1). De forma que los padres y madres que asumen rasgos más expresivos no suelen trabajar fuera de casa $(\mathrm{t}=-3.66 ; \mathrm{p}<.001)$ mientras que los que asumen rasgos más instrumentales $(\mathrm{t}=-4.64 ; \mathrm{p}<.001)$ e instrumentales-expresivos $(\mathrm{t}=-5.09$; $\mathrm{p}<.001)$ son los que trabajan fuera de sus casas.

\section{Comparación de medias del PAQ}

En relación con la escala de Expresividad, al comparar las medias de las tres muestras estudiadas, inicialmente observamos que las mayores puntuaciones en el rasgo de Expresividad, las consigue el colectivo docente, seguido de los estudiantes y de los padres y madres. Sin embargo, antes de adentrarnos en el análisis de esas diferencias tenemos que comprobar el estadístico de Levene $\left(\mathrm{F}_{(2,2674)}=14.6 ; \mathrm{p}<.001\right)$. Debido a que el nivel crítico (.000) es menor que .05, debemos rechazar la hipótesis de igualdad de varianzas y concluir que, en las muestras definidas por los tres colectivos (estudiantes, padres/madres y docentes), las varianzas de la variable Expresividad no son iguales (ver tabla 2). Puesto que no podemos asumir varianzas poblacionales iguales, debemos prestar atención a la solución propuesta por el método de Games-Howell (ver tabla 3). A este respecto podemos verificar que todos los promedios comparados difieren significativamente, siendo los y las docentes los que se adscriben a rasgos más expresivos que los y las estudiantes, y éstos más que los padres y las madres.

Tabla 2. Diferencia de medias en las escalas del PAQ en función a las tres muestras

\begin{tabular}{lccc}
\hline Muestras & Expresividad & Instrumentalidad & $\begin{array}{c}\text { Expresividad-Intrumen- } \\
\text { talidad }\end{array}$ \\
\hline Muestra de Estudiantes & 3.75 & 3.35 & 2.77 \\
Muestra de Padres y Madres & 3.59 & 3.40 & 2.53 \\
Muestra de Docentes & 3.85 & 3.43 & 2.60 \\
Prueba de Homogeneidad de & $\mathrm{F}_{(2,2674)}=$ & $\mathrm{F}_{(2,2687)}=$ & $\mathrm{F}_{(2,2644)}=$ \\
Varianzas & 14.6**$^{*}$ & $9.27^{* * *}$ & $7.71^{\star \star *}$ \\
\hline
\end{tabular}

Nota: $* \mathrm{p}<.05 ; * * \mathrm{p}<.01 ; * * * \mathrm{p}<.001$

En cuanto a la escala de Instrumentalidad, comprobamos en primer lugar la prueba de la homogeneidad de varianzas $\left(\mathrm{F}_{(2,2687)}=9.27 ; \mathrm{p}<.001\right)$. Tal y como sucedía en la escala de Expresividad, el estadístico de Levene es significativo, por tanto nos lleva también a rechazar la hipótesis de igualdad de varianzas (ver tabla 2). Ya que no es posible asumir varianzas iguales, nos servimos del método de Games-Howell que nos informa que entre las medias de los/as estudiantes y las de los padres y las madres no se producen diferencias, pero si se dan con respecto a los/as docentes que son más instrumentales (ver tabla 3). Al comparar los/as docentes con los padres y madres tampoco se producen diferencias en la escala de Instrumentalidad. Por lo tanto, son los/as docentes los que asumen rasgos más instrumentales seguidos con medias similares por el colectivo de estudiantes y de padres y madres. 
Tabla 3. Comparaciones múltiples (Games-Howell) del procedimiento ANOVA de un factor para la escala del PAQ

\begin{tabular}{|c|c|c|c|c|c|}
\hline \multirow{3}{*}{$\begin{array}{l}\text { variable } \\
\text { dependiente }\end{array}$} & (I) MUESTRA & (J) & Diferencia & Error & Sig. \\
\hline & & $\begin{array}{l}\text { MUES- } \\
\text { TRA }\end{array}$ & $\begin{array}{l}\text { de medias } \\
\quad(\mathrm{I}-\mathrm{J})\end{array}$ & típico & \\
\hline & Muestra de Estudiantes & $\mathrm{P} / \mathrm{M}$ & $.160^{*}$ & .025 & .000 \\
\hline \multirow[t]{5}{*}{ Expresividad } & & DOC & $-.098^{*}$ & .026 & .001 \\
\hline & Muestra de Padres y & EST & $-.160 *$ & .025 & .000 \\
\hline & & DOC & $-.257 *$ & .025 & .000 \\
\hline & Muestra de Docentes & EST & $.098 *$ & .026 & .001 \\
\hline & & $\mathrm{P} / \mathrm{M}$ & $.257^{*}$ & .025 & .000 \\
\hline \multirow{6}{*}{$\begin{array}{l}\text { Instrumentali- } \\
\text { dad }\end{array}$} & Muestra de Estudiantes & $\mathrm{P} / \mathrm{M}$ & -.047 & .027 & .185 \\
\hline & & DOC & $-.083 *$ & .026 & .003 \\
\hline & $\begin{array}{c}\text { Muestra de Padres y } \\
\text { Madres }\end{array}$ & EST & .045 & .027 & .185 \\
\hline & & DOC & -.036 & .028 & .396 \\
\hline & Muestra de Docentes & EST & $.083 *$ & .026 & .003 \\
\hline & & $\mathrm{P} / \mathrm{M}$ & .036 & .028 & .396 \\
\hline \multirow{6}{*}{$\begin{array}{l}\text { Instrumentali- } \\
\text { dad-Expresivi- } \\
\text { dad }\end{array}$} & Muestra de Estudiantes & $\mathrm{P} / \mathrm{M}$ & $.232 *$ & .023 & .000 \\
\hline & & DOC & $.167^{*}$ & .023 & .000 \\
\hline & $\begin{array}{c}\text { Muestra de Padres y } \\
\text { Madres }\end{array}$ & EST & $-.232 *$ & .023 & .000 \\
\hline & & DOC & $-.065^{*}$ & .024 & .015 \\
\hline & Muestra de Docentes & EST & $-.167 *$ & .023 & .000 \\
\hline & & $\mathrm{P} / \mathrm{M}$ & $.065^{*}$ & .024 & .015 \\
\hline
\end{tabular}

Nota: $*$ La diferencia entre las medias es significativa al nivel .05.; Nota: $* * * \mathrm{p}<.001$; DOC: muestra de docentes; EST: muestra de estudiantes; y P/M: muestra de padres y madres.

En la escala de Instrumentalidad-Expresividad comprobamos nuevamente que el estadístico de Levene es significativo $\left(\mathrm{F}_{(2,2644)}=7.71 ; \mathrm{p}<.001\right)$, lo que significa que no podemos asumir varianzas poblacionales iguales y rechazamos la hipótesis de igualdad de varianzas (ver tabla 2). Tomando los datos del método Games-Howell podemos constatar que todos los promedios comparados entre las tres muestras difieren significativamente, siendo los y las estudiantes los que asumen rasgos más instrumentales-expresivos que los y las docentes, y éstos más que los padres y madres (ver tabla 3).

\section{Discusión}

Tal y como han demostrado diversos estudios (Bem, 1974; Spence y Helmreich, 1978; O'Connor et al., 1978; Spence, 1993; Spence y Hall, 1996; Lameiras et al., 2006) las mujeres se adscriben a rasgos más Expresivos y los hombres asumen rasgos más Instrumentales. Nuestros resultados están en esa línea, ya que en las tres poblaciones de estudio (docentes, madres/padres, y estudiantes), las 
mujeres siguen mostrando un nivel de Expresividad superior al de los hombres, mientras que los hombres muestran mayor Instrumentalidad e Instrumentalidad-Expresividad.

La opción religiosa también juega un papel importante en relación a los rasgos estereotipados por el género, de forma que las personas que se definen con rasgos más Expresivos, en su mayoría mujeres, son además las más religiosas, esta tendencia se observa sobre todo en el grupo de madres/padres, mientras que los y las docentes que se definen con características más Instrumentales-Expresivos y los estudiantes más Instrumentales y Instrumentales-Expresivos son los que se declaran abiertamente como no creyentes.

La situación laboral de la mujer sigue poniendo de manifiesto sobre todo el grupo de padres/madres, que las más expresivas son las que asumen sus roles tradicionales de esposa y madres en el ámbito privado/doméstico y por tanto, no suelen estar integradas en el mercado laboral. Mientras que en la población docente, son los y las docentes que asumen puestos directivos los más Expresivos y más Instrumentales. Sin embargo, sería esperable que las mujeres que asumen cada vez rasgos más Instrumentales ocupen más puestos de poder (Barberá et al., 2004). Nuestros resultados confirman lo contrario: que son las profesoras más Expresivas, las que asumen en mayor medida los puestos directivos, posiblemente porque desarrollan tales cargos en un colectivo mayoritariamente femenino y se les "tolere" que puedan seguir asumiendo características expresivas que en otros puestos de trabajo las limitarían en sus roles y en sus comportamientos (Rodríguez et al, 2009).

No obstante, el objetivo principal de este estudio era determinar de quien están recibiendo los y las adolescentes las mayores influencias que cristalizan en sus rasgos de personalidad estereotipos por el género. Ya que tanto los padres y las madres como el colectivo docente, que como agentes de socialización junto con el grupo de iguales, son los encargados del desarrollo de conocimientos, de adquisición de habilidades y de competencias y de transmisión de actitudes, intereses, metas, creencias y prejuicios a sus hijos/as y/o estudiantes. Por lo tanto, la familia y la escuela, como agentes de socialización primaria y secundaria, comparten la responsabilidad de jugar un papel importante en el cuidado, formación y preparación de los más jóvenes, es decir, actúan como mediadores en la adquisición progresiva de su identidad personal y social (Bonilla y Belloch, 2000).

$\mathrm{Al}$ analizar los rasgos de personalidad de los y las docente junto con los de los padres y de las madres y comparándolos con los de los estudiantes comprobamos que el colectivo docente es el que asume las características más Expresivas y las más Instrumentales y con ello menos estereotipadas. Un dato interesante es que los y las estudiantes se definen con rasgos más Instrumentales-Expresivos, es decir, que se perciben con características asociadas tanto al rasgo Instrumental relacionado con los hombres como al rasgo Expresivo relacionado con las mujeres. En definitiva, estos resultados confirman que la influencia de sus docentes al respecto será la más efectiva para la promoción de rasgos de personalidad menos estereotipados.

Para promover un cambio profundo en el orden genérico, la escuela tiene que superar un escollo importante, que es hacer partícipe a la familia (madres y padres) de una forma más activa en las prácticas educativas con las que influir también de forma positiva en su propia formación y en su adscripción a actitudes más igualitarias y roles menos estereotipados, ya que no podemos olvidar el papel que las familias juegan a la hora de consolidar cambios de actitudes y valores y que, tal como se comprueba en este trabajo, es el colectivo que están transmitiendo los rasgos de personalidad más estereotipados por el género. Y solamente desde una colaboración conjunta familia-escuela se puede aspirar a superar las estructuras arraigadas de los estereotipos de género.

\section{Referencias}

Barberá, E. (1998). Psicología del género. Barcelona: Ariel.

Barberá, E. (2004). Perspectiva cognitiva-social: estereotipos \& esquemas de género. En E. Barberá \& I. Martínez (Eds.), Psicología y Género (pp. 55-80). Madrid: Prentice Hall.

Bem, S. (1974). The measurement of psychological androgyny. Journal of Consulting and Clinical Psychology, 42, 155-162. 
Bonilla, A., \& Belloch, A. P. (2000). Identidades, transformación de modelos sociales y su incidencia en el ámbito educativo. En J. Fernández (Eds.), Intervención en los ámbitos de la sexología y de la generología (pp.135-176). Madrid: Pirámide.

Lameiras, M. \& Rodríguez, Y. (2002). Evaluación del sexismo moderno en adolescentes. Revista de Psicología Social, 17 (2), 119-127.

Lameiras, M. \& Rodríguez, Y. (2003). Evaluación del sexismo ambivalente en estudiantes gallegos/ as. Revista de Acción Psicológica, 2 (2), 131-136.

Lameiras, M.; Rodríguez, Y.; Calado, M.; Foltz, M. \& Carrera, M. V. (2006). Expressive-Instrumental traits and sexist attitudes among Spanish university professors. Social Indicators Research, 5, $1-17$.

O'Connor, K.; Mann, D. \& Bardwick, J. (1978). Androgyny and self-esteem in the upper-middleclass: A replications of Spence. Journal of Counseling and Clinical Psychology, 46, 1168-1168.

Rodríguez, Y. (2006). Evaluación del sexismo en la comunidad educativa española. Tesis doctoral (no publicada), Universidad de Vigo.

Rodríguez, Y.; Lameiras M. \& Carrera, M.V. (2009). Análisis del espacio público versus privado en docentes no universitarios españoles. SUMMA Psicológica, 6 (1), 15-24.

Spence, J. T. (1979). Traits, roles and the concept of androgyny. En J. E. Gullahorn (Eds.), Psychology and women: In transition (pp. 95-125). Washington, Wisnton and Son.

Spence, J. T. (1984). Masculinity, femininity and gender related traits: A conceptual analysis and critique of current research. Progress in Experimental Personality Research, 23, 1-97.

Spence, J. T. (1993). Gender-related traits and gender ideology: Evidence for a Multifactorial Theory. Journal of Personality and Social Psychology, 64 (4), 624-635.

Spence, J. T. \& Buckner, C. (2000). Instrumental and expresive traits, trait stereotypes, and sexist attitudes. Psychology of Women Quarterly, 24, 44-62.

Spence, J. T. \& Hall, S. K. (1996). Children's gender-related self-perceptions, actitvity preferences, and occupational stereotypes: A test of three models of gender constructs. Sex Roles, 35 (11/12), 659-691.

Spence, J. T. \& Helmreich, R. L. (1978). Masculinity and femininity: Their psychological dimensions, correlates and antecedents. Austin: University of Texas Press.

Spence, J. T.; Helmreich, R. L. \& Stapp, J. (1974). The Personal Attributes Questionnaire: A measure of sex-role stereotypes and masculinity-feminity. JSAS Catalog of Selected Documents in Psychology, 4, 43-44.

Vergara, A. I. \& Páez, D. (1993). Revisión teórico-metodológica de los instrumentos para la medición de la identidad de género. Revista de Psicología Social, 8 (2), 132-152. 